\title{
Cognitive-behavioural therapy modestly reduces post-traumatic stress symptoms resulting from physical injury
}

Bisson Jl, Shepherd JP, Joy D, et al. Early cognitive-behavioural therapy for post-traumatic stress symptoms after physical injury. Randomised controlled trial. Br J Psychiatry 2004;184:63-9.

\section{Does cognitive-behavioural therapy shortly after physical injury reduce symptoms of post-traumatic stress?}

\section{METHODS}

$\square$

Design: Randomised controlled trial.

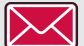

Allocation: Concealed.

Blinding: Assessors blinded to treatment

Follow up period: Thirteen months.

Setting: Hospital Accident and Emergency unit, Wales; recruitment March 1997 to February 1998.

Patients: 152 people (aged between 16 and 70 years; $43 \%$ male) physically injured, mainly by motor vehicle accident or assault, and with acute psychological distress (DSM-IV, PTSD symptom criteria from PTSD Diagnostic Scale, Hospital Anxiety and Depression Scale (HADS) score $>15$ or Impact of Event Scale (IES) score $>35$ ). Exclusions: psychiatric disorder, physical disability, illness, or cognitive deficit.

D Intervention: Cognitive behaviour therapy (CBT) involving weekly 1 hour counselling sessions with a trained psychologist for 4 weeks. Counselling started 5-10 weeks after physical injury and involved exposure therapy and cognitive restructuring. The usual care group received standard care with no psychological intervention.

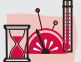

Outcomes: Primary outcome: change in IES score. Secondary outcomes: change in HADS score; PTSD symptoms assessed using clinician administered PTSD scale.

a Patient follow up: $80 \%$ CBT; $72 \%$ usual care at 13 months.

\section{MAIN RESULTS}

At 13 months, early CBT significantly reduced symptoms of PTSD measured by IES, compared with usual care (intention to treat analysis; adjusted mean difference in IES score: CBT $v$ usual care 8.4, $95 \%$ CI 2.4 to 14.4). There was no significant difference in anxiety and depression between the two groups, or in PTSD measured by the clinician administered PTSD diagnostic scale (adjusted mean difference for anxiety subscale HADS: CBT $v$ usual care $0.3,95 \%$ CI -1.0 to 1.6; adjusted mean difference for depression subscale HADS:

For correspondence: Jonathan I Bisson, Department of Liaison Psychiatry Cardiff and Vale NHS Trust, Monmouth House, University Hospital of Wales, Cardiff, UK; Bissonj|@Cardiff.ac.uk

Sources of funding: the Welsh Office of Research and Development for Health and Social Care.
CBT $v$ usual care $0.5,95 \%$ CI -0.8 to 1.9 ; adjusted mean difference for clinician administered PTSD diagnostic scale: CBT $v$ usual care -6.6 , $95 \%$ CI -13.6 to 0.4 ).

\section{CONCLUSIONS}

Psychological intervention, in the form of early cognitive-behavioural therapy, modestly reduces the symptoms of PTSD after physical injury.

\section{Commentary}

B isson's early cognitive-behavioural intervention study is an important step in delineating the necessary amount and timing of effective interventions following trauma. Whereas earlier RCTs of cognitive behavioural interventions studied individuals diagnosed with ASD, and were initiated within one month of the traumatic incident, ${ }^{1}$ this study reduced the intervention length, started treatment at a later post-trauma phase (5-10 weeks), and did not require a diagnosis of ASD for inclusion. As Bisson states, because the findings in this study are not as robust as in previous studies, it may be that four 1 hour sessions are not sufficient to prevent chronic adjustment difficulties following trauma. His intervention did not significantly reduce anxious or depressive symptoms, further clarifying possible additional treatment components that may be necessary to improve outcome. Taking a phased and modular approach to the post-trauma situation might be the most effective response to the needs of individuals, with the important caveat of taking into account the resources available.

In regards to resources, particularly in the area of disasters and mass violence, there is a strong need for efficient, effective early interventions. Recent controversy regarding the lack of effectiveness of one time interventions in preventing post-trauma psychological distress means that this type of research is critical for drawing the boundaries of effective care. Possible next steps to Bisson's intervention would be implementation in mass violence settings, traumatically bereaved populations, and different timeframes following trauma. His positive findings point to a need for larger scale training of therapists in these types of interventions, so that resources in the community will be available as needed. Also critical is finding the appropriate timing for the intervention. Recent expert consensus recommendations for interventions following mass violence suggest that structured cognitive-behavioural interventions not be implemented until secondary stressors in the environment are under sufficient control to allow the individual to focus on the intervention (usually not sooner than three weeks after incident). ${ }^{2}$ Bisson's use of this intervention suggests that it can have an impact within the 5-10 week window. In conducting such high quality randomised controlled early intervention trials, Bisson continues to be a key player in moving the field forward.

Patricia Jane Watson, PhD

Deputy for Education and Clinical Networking, National Center for PTSD, VA Regional Medical Center, White River Junction, VT, USA.

1 Bryant RA, Harvey AG, Dang ST, et al. Treatment of acute stress disorder: a comparison of cognitive-behavioral therapy and supportive counseling. J Consult Clin Psychol 1998;66:862-6.

2 Watson P. Mental health interventions following mass violence. Stresspoints 2004;12:4-5. 